\title{
BMJ Open Association between obesity-related anthropometric indices and multimorbidity among older adults in Shandong, China: a cross-sectional study
}

\author{
Jiao Zhang (D) , ${ }^{1,2,3}$ Lingzhong Xu, ${ }^{1,2,3}$ Jiajia Li, ${ }^{1,2,3}$ Long Sun (D) , ${ }^{1,2}$ Wenzhe Qin ${ }^{1,2,3}$
}

To cite: Zhang J, Xu L, Li J, et al. Association between obesity-related anthropometric indices and multimorbidity among older adults in

Shandong, China: a crosssectional study. BMJ Open 2020;10:e036664. doi:10.1136/ bmjopen-2019-036664

\section{- Prepublication history for} this paper is available online. To view these files, please visit the journal online (http://dx.doi org/10.1136/bmjopen-2019036664).

Received 27 December 2019 Revised 29 March 2020 Accepted 21 April 2020

A Check for updates

(c) Author(s) (or their employer(s)) 2020. Re-use permitted under CC BY-NC. No commercial re-use. See rights and permissions. Published by BMJ.

${ }^{1}$ School of Public Health, Cheeloo College of Medicine, Shandong University, Jinan, China

${ }^{2} \mathrm{NHC}$ Key Laboratory of Health Economics and Policy Research, Cheeloo College of Medicine, Shandong University, Jinan, China

${ }^{3}$ Center for Health Economics Experiment and Public Policy Research, Cheeloo College of Medicine, Shandong University, Jinan, China

Correspondence to

Dr Lingzhong Xu;

Izxu@sdu.edu.cn

\section{ABSTRACT}

Objectives Whether the association between obesityrelated anthropometric indices and multimorbidity differs by age among Chinese older adults (aged 65+) is unclear. We aimed to investigate the association between body mass index (BMI), waist circumference (WC) and waist-tohip ratio (WHR) with multimorbidity among the young-old (aged 65-79) and old-old (aged 80+) adults.

Design Cross-sectional population-based study. Setting Shandong province on the eastern coast of China Participants 5493 subjects aged 65 years or above. Measurements Details on sociodemographics, lifestyle characteristics and chronic conditions were collected using a structured questionnaire. The respondents were assessed with anthropometric measurements including height, weight, WC, hip circumference.

Results The overall prevalence of multimorbidity in older adults (aged 65+) was 35.2\%. The BMl-obesity, WC-obesity and WHR-obesity rates were 7.4\%, 57.5\% and $80.4 \%$, respectively. In the young-old adults (aged 65-79), the likelihood of multimorbidity was more than two times higher among the BMl-obese than the BMInormal population (OR 2.08, $95 \% \mathrm{Cl} 1.66$ to 2.60). Similar but less strong associations were found for the WC-obese and WHR-obese young-old population (OR 1.60, 95\% Cl 1.42 to 1.81 ; OR $1.31,95 \% \mathrm{Cl} 1.10$ to 1.56 , respectively). For the old-old group (aged 80+), the BMl-obese, WCobese and WHR-obese had a higher likelihood of having multimorbidity compared with the normal weight category (OR 2.10, 95\% Cl 0.96 to 4.57 ; OR $1.75,95 \% \mathrm{Cl} 1.21$ to 2.54; OR $2.15,95 \% \mathrm{Cl} 1.18$ to 3.93 , respectively). Conclusion BMI-obesity, WC-obesity and WHR-obesity were associated with a greater risk of multimorbidity, and the associations were different between the young-old and the old-old adults. These age differences need to be considered in assessing healthy body weight in old age. These findings may be vital for public health surveillance, prevention and management strategies for multimorbidity in older adults.

\section{INTRODUCTION}

Multimorbidity, defined as the simultaneous occurrence of two or more chronic conditions within an individual, is an emerging public health concern due to its increasing prevalence and impact on individual health status,

\section{Strengths and limitations of this study}

- The prevalence of multimorbidity in the young-old (aged 65-79) and the old-old (aged 80+) adults was estimated using a large number of population survey sample.

- This study examined the associations between obesity-related anthropometric indices and multimorbidity in Chinese older adults, and further explored the age-stratified patterns of the associations.

- Chronic diseases could be under-reported, which would affect the observed multimorbidity prevalence.

- The use of cross-sectional data that does not allow for exploration of causal pathways underlying the reported associations.

especially for the elderly. ${ }^{1}$ Multimorbidity affects more than half of the elderly population globally with increasing prevalence in very old persons. ${ }^{3}$ People with multimorbidity increased the burden on primary care services, ${ }^{4}$ and require higher levels of healthcare utilisation resulting in increased costs. ${ }^{56}$ As such, knowledge of risk factors associated with multimorbidity, stratified by age, will be needed to enable the delivery of high-quality healthcare adapted to patient needs. Evidence has indicated that being overweight or obese represents a major risk factor for multimorbidity. ${ }^{78}$ Moreover, obesity can exacerbate the age-related decline in physical function and lead to a higher risk of developing multimorbidity for elderly persons, thus, obesity must be identified early so that a better quality of life can be provided to this older age group. More sensitive anthropometric instruments can assist with better comprehension of the dynamics involving physiological alterations in the ageing process, thereby enhancing an obesity diagnosis and preventing the multimorbidity associated with it.

Several anthropometric indicators have been proposed to assess obesity among older 
adults. These indicators provide an indirect evaluation of body composition and a subsequent risk of chronic diseases. In studies assessing the effects of obesity on multimorbidity, the most widely used measure for obesity is body mass index (BMI).${ }^{9} 10$ However, it is known that ageing is related to poor energy regulation, reduced hormonal levels and changes in body composition, which in turn affects anthropometric measurements and risk of chronic diseases. ${ }^{11}$ BMI is criticised for its impaired validity to describe the amount of adipose tissue, especially in the abdominal region, in older adults. ${ }^{12}$ Alternative approaches are needed for older adults, and measures such as central adiposity in addition to BMI are valuable for assessing disease risk. ${ }^{13}$ Other anthropometric measurements such as waist circumference (WC) and waist-to-hip ratio (WHR) are recommended for the assessment of central obesity. Evidence indicates that there is a strong positive association between cardiovascular risk factors with measures of WC or WHR rather than BMI alone. ${ }^{14} 15$

Changes in body composition and fat distribution with ageing are poorly captured by standard anthropometric data. ${ }^{16}$ Recent evidence recommended that determination of the optimal body mass and composition for older adults should consider the age and health condition of the individual. ${ }^{17}$ Different anthropometric indices may have distinct meanings for older people of different age groups. Although some studies have explored the association between anthropometric indices and multimorbidity, ${ }^{18}{ }^{19}$ surprisingly few of them focused on older people and considered variation across different age groups. Therefore, this study aims to ascertain the associations between anthropometric indices (BMI, WC and WHR) and multimorbidity in older adults (aged 65+) and whether the associations vary among young-old (aged 65-79) versus old-old (aged 80+) adults.

\section{METHODS}

\section{Study population}

Data of this study are derived from the Survey of the Shandong Elderly Family Health Service, which was conducted by Shandong University in 2017. The detailed sampling and quality assurance measures have been described in a previously published paper. ${ }^{20}$ In brief, a three-stage, stratified, random sampling scheme was used to ensure that samples were representative of the whole population of Shandong province. In the first stage, six counties/districts were selected in Shandong province. In the second stage, 18 villages and 18 communities were selected in sampled counties/districts. In the last stage, a total of 5643 households consisting of 7088 individuals aged 60 years and above were identified. Of these, 18 did not complete the survey. In total, 7070 individuals were included in the final sample. After obtaining the verbal informed consent of respondents, all participants were interviewed individually using a structured questionnaire. There were no missing data for the dependent and independent variables, except for income $(n=26)$, which were replaced with values obtained through mean imputation. In this study, all analyses were based on 5493 subjects aged 65 years or above.

\section{Chronic conditions and multimorbidity}

A number of chronic conditions were collected via the following question: 'Have you been diagnosed with any conditions by a doctor?' The chronic diseases included hypertension, diabetes, coronary heart disease, chronic obstructive pulmonary disease, asthma, cancer and other diseases. Respondents answered 'yes' or 'no' for each question. Each condition was assessed by trained investigators separately. Multimorbidity referred to the coexistence of two or more chronic diseases in the same person. ${ }^{21}$

\section{Anthropometric measurements}

The anthropometric measurements included body height, weight, WC and hip circumference. Height was measured to the nearest $1 \mathrm{~cm}$ and weight to the nearest $1 \mathrm{~kg}$. All subjects were measured wearing light clothing without hats or shoes. BMI was computed as weight in kilograms divided by height in metres squared $(\mathrm{kg} /$ $\mathrm{m}^{2}$ ) and classified according to WHO definitions. ${ }^{22}$ The applied classifications were underweight (BMI $<18.50 \mathrm{~kg}$ / $\mathrm{m}^{2}$ ), normal weight (BMI $18.50-24.99 \mathrm{~kg} / \mathrm{m}^{2}$ ), overweight (BMI $25.00-29.99 \mathrm{~kg} / \mathrm{m}^{2}$ ) and obese (BMI $\geq 30.00 \mathrm{~kg} / \mathrm{m}^{2}$ ). WC was measured midway between the level of the iliac crest and the lowest rib and the cut-off point for abdominal obesity were chosen as a WC $>102 \mathrm{~cm}$ for males and $>88 \mathrm{~cm}$ for females. ${ }^{23}$ Hip circumference was measured from the widest part of pelvis. WHR was computed as the ratio of WC to hip circumference and the cut-off value for central obesity was considered $\geq 0.90$ for males and $\geq 0.85$ for females. ${ }^{23} \mathrm{WC}$ and WHR were used as categorical variables in the analysis.

\section{Other variables}

Sociodemographic information included age (65-79, $80+$ ), gender (male, female), residence (rural, urban), marital status (couple, single), education (no education, primary school, secondary school, high school and above), employment (employed, unemployed, retired), income ((four income quartiles: quartile 1 (q1) is the poorest and quartile 4 (q4) is the richest). Lifestyle characteristics included smoking (never-smoker, ex-smoker, current smoker), drinking (abstainer, ex-drinker, drinker), number of weekly exercise times $(\leq 1, \geq 2)$.

\section{Statistical analysis}

All data were analysed using SPSS V.24.0 (IBM). We first divided the participants into two age groups: the young-old (65-79) and the old-old (80+). Categorical variables were reported in number (percentage), and $\chi^{2}$ tests and $z$-tests were used to analyse differences in sociodemographic factors, anthropometric indices and chronic conditions across the age groups. $\chi^{2}$ tests were also used to compare the distribution of chronic conditions in different BMI, 
Table 1 Descriptive characteristics of study sample by age groups

\section{Characteristic}

Gender $\mathrm{n}(\%)$

Male

Female

Residence n (\%)

Rural Urban

Marital status $\mathrm{n}(\%)$

Couple

Single†

Education $\mathrm{n}(\%)$

No education

Primary school

Secondary school

High school and above

Employment $n$ (\%)

Employed

Unemployed

Retired

Income $\mathrm{n}(\%)$

Q1

Q2

Q3

Q4

Smoking $n(\%)$

Never-smoker

Ex-smoker

Current smoker

Drinking n (\%)

\section{Abstainer \\ Ex-drinker}

Drinker

No of weekly exercise times $n(\%)$

\begin{tabular}{l}
$\leq 1$ \\
$\geq 2$ \\
No of chronic conditions $\mathrm{n}(\%)$ \\
\hline None \\
1 \\
\hline 22 (multimorbidity) \\
BMI $\mathrm{n}(\%)$ \\
\hline Underweight \\
Normal \\
Overweight \\
Obesity \\
WC n (\%)
\end{tabular}

\section{Total}

$n=5493$

2278 (41.5)

$3215(58.5)$

$4242(77.2)$

$1251(22.8)$

4291 (78.1)

$1202(21.9)$

1808 (32.9)

2438 (44.4)

$877(16.0)$

$370(6.7)$

1421 (25.9)

$2931(53.4)$

$1141(20.8)$

1460 (26.6)

1366 (24.9)

1292 (23.5)

1375 (25.0)

3883 (70.7)

748 (13.6)

863 (15.7)

4143 (75.4)

465 (8.5)

885 (16.1)

2159 (39.3)

3334 (60.7)

1578 (28.7)

1981 (36.1)

1934 (35.2)

$204(3.7)$
$2821(51.4)$
$2064(37.6)$
$404(7.4)$

404 (7.4)

\section{Young-old \\ $n=4884$}

2017 (41.3)

2867 (58.7)

3755 (76.9)

1129 (23.1)

3959 (81.1)

925 (18.9)

$1484(30.4)$

2217 (45.4)

847 (17.3)

$336(6.9)$

1393 (28.5)

$2461(50.4)$

1030 (21.1)

1248 (25.6)

$1213(24.8)$

1171 (24.0)

1252 (25.6)

3440 (70.4)

659 (13.5)

785 (16.1)

3669 (75.1)

$414(8.5)$

801 (16.4)

801 (16.4)

$1881(38.5)$

3003 (61.5)

$1373(28.1)$

1749 (35.8)

1762 (36.1)

$165(3.4)$

$2441(50.0)$

1905 (39.0)

373 (7.6)

\section{Old-old \\ $\mathrm{n}=609$}

261 (42.9)

348 (57.1)

0.087

\section{7 (80.0)}

$122(20.0)$

$<0.001$

332 (54.5)

277 (45.5)

$<0.001$

324 (53.2)

221 (36.3)

30 (4.9)

34 (5.6)

$<0.001$

$28(4.6)$

470 (77.2)

111 (18.2)

$<0.001$

212 (34.8)

153 (25.1)

121 (19.9)

$123(20.2)$

0.104

442 (72.6)

89 (14.6)

78 (12.8)

0.244

474 (77.8)

51 (8.4)

84 (13.8)

0.001

278 (45.6)

331 (54.4)

$205(33.7) \quad<0.001$

232 (38.1)

172 (28.2)

$<0.001$

39 (6.4)

380 (62.4)

159 (26.1)

31 (5.1)

$<0.001$

Continued 
Table 1 Continued

\begin{tabular}{|c|c|c|c|c|}
\hline & Total & Young-old & Old-old & \\
\hline Characteristic & $n=5493$ & $\mathrm{n}=4884$ & $\mathrm{n}=609$ & P value* \\
\hline Normal & 2335 (42.5) & 2037 (41.7) & $298(48.9)$ & \\
\hline WHR n (\%) & & & & 0.832 \\
\hline Normal & $855(15.6)$ & $762(15.6)$ & 93 (15.3) & \\
\hline
\end{tabular}

Young-old means aged 65-79, old-old means aged 80 or over.

${ }^{*} \chi^{2}$.

†Single, not married/divorced/widowed or others.

BMI, body mass index; WC, waist circumference; WHR, waist-to-hip ratio.

WC and WHR categories among the young-old and oldold adults. The associations between each anthropometric measure (BMI, WC and WHR as independent variables) and multimorbidity were estimated via logistic regression, providing ORs and 95\% CIs. In all analyses, BMI, WC and WHR were modelled as categorical variables. The regression models were adjusted for gender, residence, marital status, education, employment, income, smoking, drinking and number of weekly exercise times.

\section{Patient and public involvement}

No patients or member of the public were involved in the conception, design and planning of this study.

\section{RESULTS}

\section{Characteristics of participants}

Table 1 shows the age-stratified characteristics of the study sample. In total, 5493 elderly people were enrolled, $11.1 \%$ of them were aged $80+, 58.5 \%$ were female and most of them lived in a rural area $(77.2 \%)$ and were couples $(78.1 \%)$. As expected, in contrast to the young-old, most of the old-old were unemployed, had a lower level of education and income, and did less weekly exercise. As for chronic conditions, $28.7 \%$ of older adults reported no chronic disease, $36.1 \%$ reported having one chronic condition and $35.2 \%$ reported multimorbidity. The prevalence of multimorbidity in the young-old was higher than that in the old-old ( $36.1 \%$ vs $28.2 \%$ ). Regarding obesityrelated anthropometric indices, the young-old were more likely to be BMI-obese and WC-obese compared with the old-old $(7.6 \%$ vs $5.1 \%$ and $58.3 \%$ vs $51.1 \%)$. No difference was observed in the distribution of WHR-obesity between the two age groups.

\section{The prevalence of multimorbidity by BMI, WC and WHR categories}

The prevalence of multimorbidity according to BMI, WC and WHR categories is shown in table 2. The prevalence of multimorbidity was $49.3 \%, 65.2 \%$ and $36.7 \%$ among BMI-obese, WC-obese and WHR-obese population, respectively. The increase in multimorbidity prevalence which was mirrored by an increase in BMI (overweight and obesity), both for the young-old and old-old but more pronounced in young-old adults. Also, those with central obesity (WC-obesity and WHR-obesity) reported a higher mean number of chronic conditions and a higher prevalence of multimorbidity, both in the young-old and old-old population.

\section{Association of BMI, WC and WHR with multimorbidity}

Table 3 shows, for each age group separately, results of the logistic regressions that estimated the association of BMI, WC and WHR with multimorbidity. Overall, the BMIobese or central obese had a higher likelihood of having a chronic condition compared with those normal weight. In young-old adults, after multivariable adjustment, the likelihood of multimorbidity was still more than two times higher among the BMI-obese than the BMI-normal population (OR 2.08, 95\% CI 1.66 to 2.60). Similar but less strong associations were found for the WC and WHR young-old populations (OR 1.60, 95\% CI 1.42 to 1.81 ; OR $1.31,95 \%$ CI 1.10 to 1.56 , respectively). With regard to the old-old, BMI-obesity was no longer associated with multimorbidity whereas WC-obesity and WHR-obesity remained positively associated with multimorbidity (OR $1.75,95 \%$ CI 1.21 to 2.54 ; OR $2.15,95 \%$ CI 1.18 to 3.93 , respectively). An analysis of potential gender differences in obesity measures and multimorbidity was undertaken, however, it was found that men and women were similar in both outcomes.

\section{DISCUSSION}

This study was performed to assess the association between obesity-based anthropometric indices and multimorbidity in older adults. To our knowledge, this is the first study to examine determine whether the association varied across two age groups among older adults $(65+)$ in China. We controlled for many confounders, including sociodemographic, socioeconomic characteristics and living habits.

The prevalence of multimorbidity was $35.2 \%$ in the sample of Shandong, China, with a higher prevalence of multimorbidity in the young-old (aged 65-79) than that in the old-old (aged 80+). Numerous studies regarding 





Table 3 Association between BMI, WC and WHR status and multimorbidity in young-old $(n=4884)$ and old-old $(n=609)$ adults

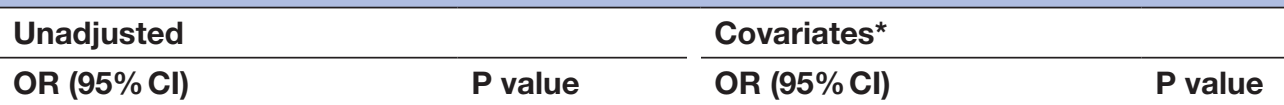

\section{Young-old}

BMI

\begin{tabular}{|c|c|c|c|c|}
\hline Normal & Ref & & Ref & \\
\hline Underweight & 0.71 (0.50 to 1.03$)$ & 0.071 & 0.70 (0.48 to 1.02$)$ & 0.060 \\
\hline Overweight & 1.55 (1.36 to 1.75$)$ & $<0.001$ & $1.48(1.30$ to 1.68$)$ & $<0.001$ \\
\hline \multicolumn{5}{|l|}{ WC } \\
\hline Normal & Ref & & Ref & \\
\hline \multicolumn{5}{|l|}{ WHR } \\
\hline Normal & Ref & & Ref & \\
\hline Central obesity & $1.50(1.26$ to 1.77$)$ & 0.001 & $1.31(1.10$ to 1.56$)$ & 0.003 \\
\hline \multicolumn{5}{|l|}{ Old-old } \\
\hline \multicolumn{5}{|l|}{$\mathrm{BMI}$} \\
\hline Overweight & $1.28(0.85$ to 1.93$)$ & 0.233 & $1.23(0.81$ to 1.88$)$ & 0.335 \\
\hline Obesity & 2.08 (0.98 to 4.40$)$ & 0.056 & $2.10(0.96$ to 4.57$)$ & 0.063 \\
\hline \multicolumn{5}{|l|}{ WC } \\
\hline Normal & Ref & & Ref & \\
\hline Central obesity & 1.82 (1.27 to 2.61$)$ & 0.001 & 1.75 (1.21 to 2.54$)$ & 0.003 \\
\hline \multicolumn{5}{|l|}{ WHR } \\
\hline Normal & Ref & & Ref & \\
\hline Central obesity & 2.09 (1.18 to 3.69$)$ & 0.012 & 2.15 (1.18 to 3.93$)$ & 0.013 \\
\hline
\end{tabular}

*BMI, WC and WHR each separately adjusted for gender, residence, marital status, education, employment, income, smoking, drinking, number of weekly exercise times.

BMI, body mass index; WC, waist circumference; WHR, waist-to-hip ratio.

the prevalence of multimorbidity have been conducted in China. ${ }^{24-26}$ These studies included different data sources, targeted age groups, definitions of multimorbidity and number of chronic conditions integrated in the analyses. Consequently, the estimated prevalence of multimorbidity varied. A previous systematic review reported that the overall prevalence of multimorbidity among older adults (aged 60+) ranged widely from $6.4 \%$ to $86.9 \%$ in China. ${ }^{27}$ Regarding to the difference between young-old and old-old, a multicountry study showed that multimorbidity prevalence was high in older adults (aged $50+$ ) across countries, and increased with age, although the rate of increase in the oldest age groups $(80+)$ was modest at best in most countries with declines observed in some countries. ${ }^{28}$ Moreover, evidence indicates that life expectancy is substantially lower in people with multimorbidity $^{29} 30$; the most old-old people with multimorbidity may have already died, while those with less chronic diseases live longer, which may result in lower prevalence of multimorbidity in the old-old than that in the young-old. It was shown that survey chronic conditions data from patients may not be entirely concordant with medical record documentation. ${ }^{31}$ However, the difference attenuates with age, and the prevalence is similar in both data sources for elderly patients. ${ }^{32}$ The prevalence of multimorbidity reported in this study, despite the difference between this study and others, is consistent with the literature, indicating that multimorbidity is a common occurrence in older population and should receive more attention, especially in the young-old adults.

Multimorbidity more often occurred in subjects with BMI-obesity/central obesity than in those with normal weight, which was aligned with previous reports that obesity was an important risk factor for chronic diseases, which can lead to multimorbidity. ${ }^{33}$ With regard to the positive association between obesity and multimorbidity, our results were consistent with those of Jovic et $a l,{ }^{19}$ who reported that obesity was associated with increased prevalence of multimorbidity. Other research also showed that obesity was independently associated with the occurrence 
of multimorbidity in adults from six low-income and middle-income countries (China, India, Mexico, Russia, South Africa and Ghana). ${ }^{18}$ In these studies, however, obesity was assessed by BMI alone. Our results indicated that WC/WHR central obesity was also correlated with increased risk of multimorbidity. This may have important implications for the potential to delay onset of multimorbidity through earlier and more effective intervention on BMI-obesity and central obesity.

Furthermore, we found some different pattern between the young-old and old-old adults. More specifically, in young-old adults, BMI-obesity and central-obesity were associated with increased multimorbidity risk. In the oldold, however, a positive association was observed only in the case of central obesity. Our findings support the contention that abdominal obesity is associated with increased risk of multimorbidity independently of potential confounding factors. ${ }^{35}$ WC or WHR may be the better screening measure for chronic conditions than other anthropometric indicators. For example, Wang et $a l^{36}$ reported that WC was a better measure of central obesity than WHR for predicting the risk of type 2 diabetes, while other studies showed that among the anthropometric indices of obesity, WHR was better than BMI, bodyweight and WC in predicting chronic conditions, such as chronic kidney disease ${ }^{37}$ and type 2 diabetes $^{38}$ in the elderly. Our study extends the previous findings because we found that both WC and WHR are more sensitive to screening for multimorbidity than BMI, especially in old-old (aged $80+$ ) adults. Another possible reason for such variations in the associations of anthropometric indices with multimorbidity is that body composition changes as age advances, and BMI cannot accurately describe body fat distribution for those in old-old age compared with other indicators; this deserves further exploration. Based on these findings, multiple obesity-related indicators should be used to predict multimorbidity in the elderly population in order to avoid biases caused by the use of a single indicator. We recommend that WC and WHR are more important and necessary indicators.

There are still some limitations in this study. First, chronic diseases could be under-reported, which would affect the observed multimorbidity prevalence. Second, other anthropometric indicators, such as waist-to-height ratio and hip index, were not considered in this study. Therefore, further studies are required in the future. Third, because our study was a cross-sectional design, causality cannot be inferred. Despite these limitations, this study elaborates on the available evidence on multimorbidity in the Chinese older population.

\section{CONCLUSIONS}

In conclusion, the prevalence of multimorbidity among Chinese aged $\geq 65$ years was high and varied by age. The associations between BMI-obesity and WC, WHR central obesity and multimorbidity were different between the young-old and old-old adults. Further research on investigating the underlying mechanisms linking BMIobesity/central obesity and multimorbidity among older adults should consider different age subgroups. Our findings also highlight the importance of using multiple body mass indicators to predict chronic conditions in the older population.

Contributors JZ performed the statistical analysis and drafted the initial manuscript, $L X$ and JL provided the concept and design of study. LS and WQ was involved in the analysis and interpretation of data and revised the manuscript for important intellectual content. All authors read and approved the final manuscript.

Funding Our work was supported by two grants of National Natural Science Foundation of China (grant numbers: 71673169 and 71673170) and NHC Key Laboratory of Health Economics and Policy Research (Shandong University) (NHC-HEPR2019015).

Competing interests None declared.

Patient and public involvement Patients and/or the public were not involved in the design, or conduct, or reporting, or dissemination plans of this research.

Patient consent for publication Not required.

Ethics approval The Ethical Committee of Shandong University School of Public Health approved the study protocol (No. 20170110).

Provenance and peer review Not commissioned; externally peer reviewed.

Data availability statement Data are available on reasonable request. Data are available on reasonable request to corresponding author (email: Izxu@sdu.edu.cn).

Open access This is an open access article distributed in accordance with the Creative Commons Attribution Non Commercial (CC BY-NC 4.0) license, which permits others to distribute, remix, adapt, build upon this work non-commercially, and license their derivative works on different terms, provided the original work is properly cited, appropriate credit is given, any changes made indicated, and the use is non-commercial. See: http://creativecommons.org/licenses/by-nc/4.0/.

\section{ORCID iDs}

Jiao Zhang http://orcid.org/0000-0002-2881-7755

Long Sun http://orcid.org/0000-0002-3070-8427

\section{REFERENCES}

1 Kuzuya M. Era of geriatric medical challenges: multimorbidity among older patients. Geriatr Gerontol Int 2019;19:699-704.

2 Vetrano DL, Calderón-Larrañaga A, Marengoni A, et al. An international perspective on chronic multimorbidity: approaching the elephant in the room. J Gerontol A Biol Sci Med Sci 2018;73:1350-6.

3 Marengoni A, Angleman S, Melis R, et al. Aging with multimorbidity: a systematic review of the literature. Ageing Res Rev 2011;10:430-9.

4 Violán C, Foguet-Boreu Q, Roso-Llorach A, et al. Burden of multimorbidity, socioeconomic status and use of health services across stages of life in urban areas: a cross-sectional study. BMC Public Health 2014;14:530.

5 Lee JT, Hamid F, Pati S, et al. Impact of noncommunicable disease multimorbidity on healthcare utilisation and out-of-pocket expenditures in middle-income countries: cross sectional analysis. PLoS One 2015;10:e0127199-107.

6 Agborsangaya CB, Lau D, Lahtinen M, et al. Health-Related quality of life and healthcare utilization in multimorbidity: results of a crosssectional survey. Qual Life Res 2013;22:791-9.

7 Kivimäki M, Kuosma E, Ferrie JE, et al. Overweight, obesity, and risk of cardiometabolic multimorbidity: pooled analysis of individual-level data for 120813 adults from 16 cohort studies from the USA and Europe. Lancet Public Health 2017;2:e277-85.

8 Samper-Ternent R, Al Snih S. Obesity in older adults: epidemiology and implications for disability and disease. Rev Clin Gerontol 2012;22:10-34.

9 Lebenbaum M, Zaric GS, Thind A, et al. Trends in obesity and multimorbidity in Canada. Prev Med 2018;116:173-9.

10 Booth HP, Prevost AT, Gulliford MC. Impact of body mass index on prevalence of multimorbidity in primary care: cohort study. Fam Pract 2014;31:38-43.

11 Goh LGH, Dhaliwal SS, Welborn TA, et al. Anthropometric measurements of general and central obesity and the prediction of 
cardiovascular disease risk in women: a cross-sectional study. BMJ Open 2014;4:e004138.

12 Chang S-H, Beason TS, Hunleth JM, et al. A systematic review of body fat distribution and mortality in older people. Maturitas 2012;72:175-91.

13 Adab $\mathrm{P}$, Pallan M, Whincup $\mathrm{PH}$. Is BMI the best measure of obesity? BMJ 2018;360:k1274

14 Song X, Jousilahti P, Stehouwer CDA, et al. Comparison of various surrogate obesity indicators as predictors of cardiovascular mortality in four European populations. Eur J Clin Nutr 2013;67:1298-302.

15 De Nicola L, Conte G. Waist: hip ratio is a better predictor of cardiovascular risk than BMI in patients with moderate CKD. Nat Clin Pract Nephrol 2008;4:592-3.

16 Seidell JC, Visscher TL. Body weight and weight change and their health implications for the elderly. Eur J Clin Nutr 2000;54 Suppl 3:S33-9.

17 Porter Starr KN, Bales CW. Excessive body weight in older adults. Clin Geriatr Med 2015;31:311-26.

18 Agrawal S, Agrawal PK. Association between body mass index and prevalence of multimorbidity in low-and middle-income countries: a cross-sectional study. Int J Med Public Health 2016;6:73-83.

19 Jovic D, Marinkovic J, Vukovic D. Association between body mass index and prevalence of multimorbidity: a cross-sectional study. Public Health 2016;139:103-11.

20 Zhang J, Xu L, Li J, et al. Loneliness and health service utilization among the rural elderly in Shandong, China: a cross-sectional study. Int J Environ Res Public Health 2018;15:1468.

21 World Health Organization. Multimorbidity: World Health organization, 2016. Available: https://apps.who.int/iris/bitstream/handle/10665/ 252275/9789241511650-eng.pdf?sequence=1 [Accessed 30 Aug 2019].

22 World Health Organization. Bmi classification: World Health organization, 2004. Available: http://apps.who.int/bmi/index.jsp? introPage=intro_3.html [Accessed 26 Aug 2019].

23 World Health Organization. Waist circumference and waist-hip ratio:Report of a WHO expert consultation, 2011. Available: https://www.who.int/nutrition/publications/obesity/WHO_report waistcircumference_and_waisthip_ratio/en/ [Accessed 26 Aug 2019].

24 Zhang R, Lu Y, Shi L, et al. Prevalence and patterns of multimorbidity among the elderly in China: a cross-sectional study using national survey data. BMJ Open 2019;9:e024268.

25 Yao S-S, Cao G-Y, Han L, et al. Prevalence and patterns of multimorbidity in a nationally representative sample of older
Chinese: results from CHARLS. J Gerontol A Biol Sci Med Sci 2019. doi:10.1093/gerona/glz185. [Epub ahead of print: 13 Aug 2019].

$26 \mathrm{Gu}$ J, Chao J, Chen W, et al. Multimorbidity in the communitydwelling elderly in urban China. Arch Gerontol Geriatr 2017;68:62-7.

$27 \mathrm{Hu} \mathrm{X,} \mathrm{Huang} \mathrm{J,} \mathrm{Lv} \mathrm{Y,} \mathrm{et} \mathrm{al.} \mathrm{Status} \mathrm{of} \mathrm{prevalence} \mathrm{study} \mathrm{on}$ multimorbidity of chronic disease in China: systematic review. Geriatr Gerontol Int 2015;15:1-10.

28 Garin N, Koyanagi A, Chatterji S, et al. Global multimorbidity patterns: a cross-sectional, population-based, multi-country study. J Gerontol A Biol Sci Med Sci 2016;71:205-14.

29 DuGoff EH, Canudas-Romo V, Buttorff C, et al. Multiple chronic conditions and life expectancy: a life table analysis. Med Care 2014;52:688-94.

30 , Di Angelantonio E, Kaptoge S, et al, Emerging Risk Factors Collaboration. Association of cardiometabolic multimorbidity with mortality. JAMA 2015;314:52-60.

31 Corser W, Sikorskii A, Olomu A, et al. "Concordance between comorbidity data from patient self-report interviews and medical record documentation". BMC Health Serv Res 2008;8:85

32 Violán C, Foguet-Boreu Q, Hermosilla-Pérez E, et al. Comparison of the information provided by electronic health records data and a population health survey to estimate prevalence of selected health conditions and multimorbidity. BMC Public Health 2013;13:251

33 Diederichs C, Berger K, Bartels DB. The measurement of multiple chronic diseases--a systematic review on existing multimorbidity indices. J Gerontol A Biol Sci Med Sci 2011;66:301-11.

34 Agborsangaya $\mathrm{CB}$, Ngwakongnwi $\mathrm{E}$, Lahtinen $\mathrm{M}$, et al. Multimorbidity prevalence in the general population: the role of obesity in chronic disease clustering. BMC Public Health 2013;13:1161.

35 An KO, Kim J. Association of sarcopenia and obesity with multimorbidity in Korean adults: a nationwide cross-sectional study. J Am Med Dir Assoc 2016;17:960.e1-7.

36 Wang Y, Rimm EB, Stampfer MJ, et al. Comparison of abdominal adiposity and overall obesity in predicting risk of type 2 diabetes among men. Am J Clin Nutr 2005;81:555-63.

37 Chou C-Y, Lin C-H, Lin C-C, et al. Association between waist-tohip ratio and chronic kidney disease in the elderly. Intern Med $J$ 2008;38:402-6.

38 Cheng C-H, Ho C-C, Yang C-F, et al. Waist-to-hip ratio is a better anthropometric index than body mass index for predicting the risk of type 2 diabetes in Taiwanese population. Nutr Res 2010;30:585-93. 